\title{
An Expanding Ring in the Gaseous Disk of NGC 6181?
}

J. Boulesteix ${ }^{(1)}$, O. K. Sil'chenko(2), A. V. Zasov ${ }^{(2)}$, A. N. Burenkov ${ }^{(3)}$

(1) Observatoire de Marseille, France

(2) Sternberg Astronomical Institute, Moscow, Russia

(3) Special Astrophysical Observatory, Nizhnij Arkhyz, Russia

Abstract. The Sc-galaxy NGC 6181 was observed at the $6 \mathrm{~m}$ telescope with the Fabry-Perot interferometer CIGALE in $\boldsymbol{H}_{\alpha}$ emission. A ring-like zone of strong radial gas motions at the radius of $1.8 \mathrm{kpc}$ was found.

The first kinematical investigation of NGC 6181 was presented by Burbidge et al. (1965). From long-slit observations they found radial velocity profiles to be quite asymmetrical and concluded that NGC 6181 is intermediate between a barred and a normal spiral. Our long-slit observations of NGC 6181 at the $6 \mathrm{~m}$ telescope confirmed the asymmetry of one-dimensional velocity profiles (Afanasiev et al., 1992). The necessity of obtaining two-dimensional velocity field became evident for us.

\section{Observations}

The two-dimensional velocity field of NGC 6181 was obtained at the $6 \mathrm{~m}$ telescope on September 24, 1993, by using a scanning Fabry-Perot interferometer with IPCS system. A narrow-band interference filter $\left(\lambda_{c}=6620 \mathrm{~A}, F W H M=10\right.$ A) selected the spectral range of the redshifted $H_{\alpha}$.

The seeing was better than 1.5 arcsec. The resulting linear scale and spectral resolution were $0.7 \mathrm{arcsec} / \mathrm{px}$ and $40 \mathrm{~km} / \mathrm{s}$. A reduction of observational data was done by using standard methods and the software ADHOC (Observatoire de Marseille).

\section{Results}

The fit of a circular rotation model was made for the whole velocity map up to the radius of $12 \mathrm{kpc}$. Inclination and position angle of the disk for the best fit are: $i=56^{\circ}, P A_{0}=173^{\circ}$. If we compare these values with the outer isophote parameters - $i=61^{\circ}$ (Bottinelli et al., 1984) and $P A_{0}=175^{\circ}$ (Nilson 1973) - it becomes clear that the most part of the galaxy disk is in circular rotation. But the dynamical center does not coincide with the brightest central $\boldsymbol{H}_{\boldsymbol{\alpha}}$-region. If the brightest point of $H_{\alpha}$ on the long-slit spectrograms was erroneously taken as the nucleus, it may explain partially a strong asymmetry of velocity profiles observed earlier.

We have subtracted the regular rotation velocities from the observed velocity map; the residuals are shown in Fig.1. The main location of non-circular 


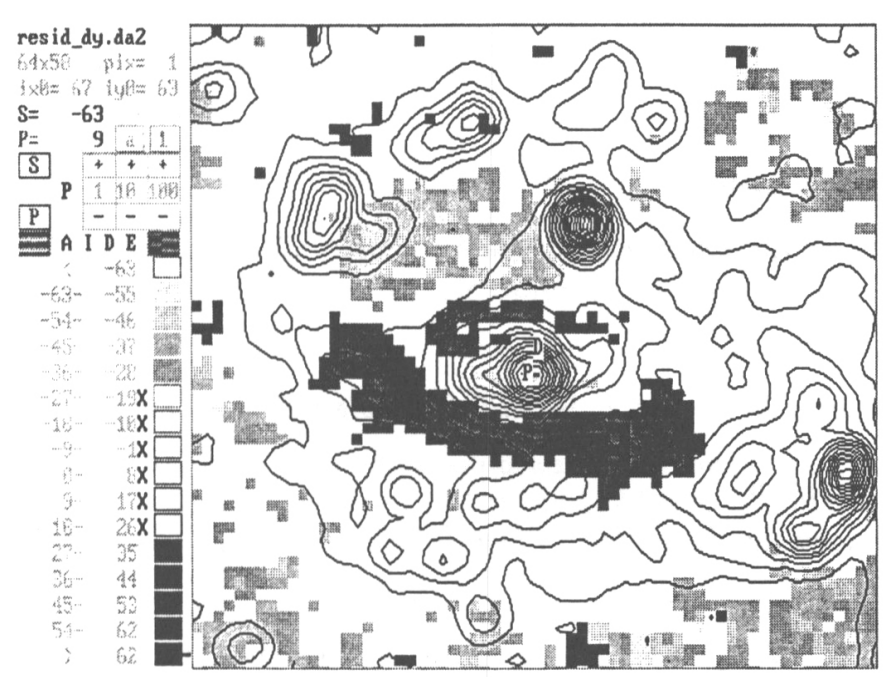

Figure 1. The residual velocities after circular rotation subtraction. The range $0 \pm 26 \mathrm{~km} / \mathrm{s}$ is shown as white, the larger residuals are greyscaled. The isophotes show $\boldsymbol{H}_{\alpha}$-emission intensity. The letters mark the positions of centers: $\mathrm{P}$ - photometric $\left(\boldsymbol{H}_{\alpha}\right)$ one, D - dynamical one

motions represents a continuous resolved zone around the center that, being deprojected onto the plane of the galaxy, looks like three quarters of a ring with a radius of 11 arcsec. The eastern half of the ring has positive residual velocities up to $60 \mathrm{~km} / \mathrm{s}$, the western part has negative ones, from -30 to $-40 \mathrm{~km} / \mathrm{s}$. The fact that the switching of residual velocity sign takes place at the line of nodes implies that the residual velocities are mostly radial ones. Assuming that the galaxy has trailing spiral arms, we derive a radial gas outflow velocity of 100 $\mathrm{km} / \mathrm{s}$.

In the very center of the galaxy the emission-line profiles are complex and obviously multi-component. This fact, together with a turn of the dynamical major axis by $30^{\circ}$ in the center, gives evidence for a small bar. The ring-like zone of strong radial gas motions and the bar may be related. Further investigation, both observational and theoretical, is necessary for this galaxy.

\section{References}

Afanasiev, V. L., Burenkov, A. N., Zasov, A. V., and Sil'chenko, O. K. 1992. $A Z h, 69,19$

Bottinelli, L., Gouguenheim, L., Paturel, G., and de Vaucouleurs, G. 1984. $A \mathcal{E} A S, \mathbf{5 6}, 381$

Burbidge, E. M., Burbidge, G. R., and Prendergast, K. H. 1965. ApJ, 142, 649

Nilson, P. 1973. Uppsala General Catalogue of Galaxies. Uppsala: Obs. Press 